\title{
Effect of brain-computer interface training based on non-invasive electroencephalography using motor imagery on functional recovery after stroke - a systematic review and meta-analysis
}

Antje Kruse ${ }^{1,2}$, Zorica Suica ${ }^{3}$, Jan Taeymans ${ }^{1,4}$ and Corina Schuster-Amft ${ }^{3,5,6^{*}}$ (D)

\begin{abstract}
Background: Training with brain-computer interface $(\mathrm{BCl})$ technology in the rehabilitation of patients after a stroke is rapidly developing. Numerous RCT investigated the effects of $\mathrm{BCl}$ training (BCIT) on recovery of motor and brain function in patients after stroke.

Methods: A systematic literature search was performed in Medline, IEEE Xplore Digital Library, Cochrane library, and Embase in July 2018 and was repeated in March 2019. RCT or controlled clinical trials that included BCIT for improving motor and brain recovery in patients after a stroke were identified. Data were meta-analysed using the random-effects model. Standardized mean difference (SMD) with 95\% confidence $(95 \% \mathrm{Cl})$ and $95 \%$ prediction interval $(95 \% \mathrm{Pl})$ were calculated. A meta-regression was performed to evaluate the effects of covariates on the pooled effect-size.

Results: In total, 14 studies, including 362 patients after ischemic and hemorrhagic stroke (cortical, subcortical, 121 females; mean age 53.0+/- 5.8; mean time since stroke onset 15.7+/- 18.2 months) were included. Main motor recovery outcome measure used was the Fugl-Meyer Assessment. Quantitative analysis showed that a $\mathrm{BCl}$ training compared to conventional therapy alone in patients after stroke was effective with an SMD of 0.39 (95\% Cl: 0.17 to $0.62 ; 95 \% \mathrm{PI}$ of 0.13 to 0.66$)$ for motor function recovery of the upper extremity. An SMD of 0.41 ( $95 \% \mathrm{Cl}:-0.29$ to 1.12) for motor function recovery of the lower extremity was found. $\mathrm{BCl}$ training enhanced brain function recovery with an SMD of 1.11 (95\%Cl: 0.64 to 1.59; $95 \% \mathrm{PI}$ ranging from 0.33 to 1.89). Covariates such as training duration, impairment level of the upper extremity, and the combination of both did not show significant effects on the overall pooled estimate.

(Continued on next page)
\end{abstract}

\footnotetext{
* Correspondence: c.schuster@reha-rhf.ch

${ }^{3}$ Research Department, Reha Rheinfelden, Salinenstrasse 98, 4310 Rheinfelden, Switzerland

${ }^{5}$ Department of Engineering and Information Technology, Pestalozzistrasse 20, 3401 Burgdorf, Switzerland

Full list of author information is available at the end of the article
}

(C) The Author(s). 2020 Open Access This article is licensed under a Creative Commons Attribution 4.0 International License, which permits use, sharing, adaptation, distribution and reproduction in any medium or format, as long as you give appropriate credit to the original author(s) and the source, provide a link to the Creative Commons licence, and indicate if changes were made. The images or other third party material in this article are included in the article's Creative Commons. licence, unless indicated otherwise in a credit line to the material. If material is not included in the article's Creative Commons licence and your intended use is not permitted by statutory regulation or exceeds the permitted use, you will need to obtain permission directly from the copyright holder. To view a copy of this licence, visit http://creativecommons.org/licenses/by/4.0/ The Creative Commons Public Domain Dedication waiver (http://creativecommons.org/publicdomain/zero/1.0/) applies to the data made available in this article, unless otherwise stated in a credit line to the data. 


\begin{abstract}
(Continued from previous page)
Conclusion: This meta-analysis showed evidence that $\mathrm{BCl}$ training added to conventional therapy may enhance motor functioning of the upper extremity and brain function recovery in patients after a stroke. We recommend a standardised evaluation of motor imagery ability of included patients and the assessment of brain function recovery should consider neuropsychological aspects (attention, concentration). Further influencing factors on motor recovery due to $\mathrm{BCI}$ technology might consider factors such as age, lesion type and location, quality of performance of motor imagery, or neuropsychological aspects.
\end{abstract}

Trial Registration: PROSPERO registration: CRD42018105832.

Keywords: Stroke, Motor imagery, Brain computer interface training, Rehabilitation, Systematic review, Meta-analysis

\section{Background}

The prevalence of stroke and the number of patients living with its consequences are increasing [1]. Advances in medical management of patients with stroke over the past decade have significantly reduced mortality, however, one third of the annually 16 million patients worldwide remain disabled [2]. Thus, more efficient stroke rehabilitation strategies are needed [1].

Brain-machine interfaces or brain-computer interfaces (BCI) in particular, are novel technologies enabling interaction with an individual's environment through brain signals [3]. This technology records physiological measures of mental processes directly from the brain and decodes them into control signals that can operate external devices or a computer [4]. In recent years, such systems have been further developed to help patients after a stroke to regain their mobility and support motor function recovery by inducing activity-dependent brain plasticity [5]. Different types of input signals were used in development of clinical practice and research projects to control BCI. About 76.1\% off all BCI Research Award submissions during the year 2015 used electroencephalography (EEG) based systems measuring motor imagery (MI) evoked potentials [5]. Other devices using non-invasive sensor systems are: Magnetoencephalography, functional Near-Infrared Spectroscopy and functional Magnetic Resonance Imaging. Invasive methods like subdural electrocorticography using action potential, intracortical local field potentials, and epidural field potentials [6] represent other possibilities for input signal sensors.

Motor imagery (MI) can be defined as the mental representation of movement without any overt body movement $[6,7]$. This involves a visual or mental representation with or without a kinaesthetic feeling [6]. It is a complex cognitive operation, which is self-generated from the patients [8]. MI offers a unique opportunity for patients after a stroke, who are unable to move their extremities by attempting to stimulate the brain regions responsible for motor movement. MI combined with conventional therapy may improve outcomes more than conventional therapy only $[9,10]$. BCI training (BCIT) systems can use EEG signals from MI performance with sensory real-time feedback and decode these signals to enable patients to direct devices such as personal computers, wheelchairs, robots, and prosthetic devices including exoskeletons. Some studies have investigated the efficacy of applying BCIT using MI on motor recovery for patients with subacute or chronic stroke with hemiparesis [3, 11-29]. Although these studies demonstrated a significant effect on recovery, the studies were flawed by low number of participants, low number of training sessions and/or a lack of follow-up assessments [3, 11-29].

We assumed that the efficacy of BCIT added to conventional therapy on motor function recovery of upper and lower extremity and brain function recovery is more beneficial than conventional therapy alone in patients after a stroke. The overall aim of our systematic review was to summarize the evidence from RCTs comparing BCIT to other therapy methods in patients after a stroke focusing on recovery of motor and brain function. The systematic review and meta-analyses aimed to answer the question: What is the effect of a brain-computer interfaces-based training with non-invasive EEG using MI on motor function and brain function recovery in patients after a stroke? We hypothesized that BCI training added to conventional therapy for motor function recovery of upper and lower extremity outperforms training without BCI technology. Furthermore, brain function recovery was evaluated as an objective parameter to indicate structural reorganisation in brain activity. Authors described different methods in their studies how they measured brain function recovery. Physiological measures that provide dynamic physiological information about brain function allow researchers to measure the contributions of various brain structures to specific psychological processes while participants complete motor or cognitive tasks. Functional brain measurement techniques [30] can measure increased regional blood flow, changes in oxygenation concentration during neural activities, glucose level, and brain cells communication by electrical impulses reflected by fluctuating lines in EEG recordings. Structural brain measurements [31] allow to examine the brain's anatomical structure and to evaluate anatomical references, 
including tissue atrophy and white matter integrity. White matter integrity of premotor-motor connections is associated with motor output in chronic stroke patients [32].

\section{Methods}

The review protocol was prepared according to the preferred reporting items for systematic review and metaanalysis protocols statements (PRISMA-P) [33] and was registered with the International Prospective Register of Systematic Reviews (PROSPERO, Registration number: CRD42018105832). The systematic review report was written following the Preferred Reporting Items for Systematic review and Meta-Analysis (PRISMA) guidelines and the PRISMA checklist [34].

\section{Search strategy, selection criteria and process}

A systematic literature search was performed in Medline via EBSCO-Host surface, IEEE Xplore Digital Library, Cochrane library, and Embase. The US National Institute of Health's ongoing trial register 'clinical.trials.gov'was searched to check for ongoing studies and unpublished reports. The search was carried out in July 2018 by a research librarian and was repeated for an update by the first author in March 2019. The search terms, strategy, and selection criteria are based on the PICOS system and were adapted for each database (see Additional file 1). Detailed inclusion criteria are listed in Table 1.

Our literature search was not restricted to any languages. All abstracts were available in English. None of the selected abstracts or full texts were excluded due to language. Studies were also excluded if they described interventions with animals or when full texts from authors were not available or were not formally peer reviewed. To decrease the risk of missing relevant studies, reference lists in the included studies were screened. Two independent reviewers (AK, ZS) screened for titles, abstracts and citations after removing duplicates from the eligible studies. To examine the agreement between the two reviewers (AK, ZS) in the pre-testing phase, 10 $\%$ of all studies were randomly selected and screened by both reviewers to check for congruence in selection. After screening of the titles and abstracts, full texts were evaluated. In case of disagreement, a third reviewer was consulted (CS) to decide on inclusion or exclusion of the study. Cohen's Kappa statistic was used to evaluate the reviewer agreement [35].

\section{Data extraction}

One researcher (AK) extracted the data from the selected studies. Data were manually entered into a Microsoft Excel (Version 14.0, 2010, Microsoft Corp., Redmond, California, USA) spreadsheet. The data extraction procedure was pilot tested on three studies, then reviewed, discussed and adjusted in accordance between the two reviewers (AK, ZS). After the pilot test, AK performed the complete data extraction and the second reviewer (ZS) crosschecked all extracted data. If needed, a consensus meeting and discussion resolved disagreement. A detailed description of the data collection process and a complete overview of extracted data are provided in Additional file 2. In case of incomplete data (e.g. only graphical presentations, missing $p$-values) in the selected studies, the corresponding authors were contacted to obtain the missing details.

\section{Assessment of risk of bias and GRADE}

Two reviewers (AK, ZS) assessed risk of bias within studies using the Cochrane Collaboration risk of bias (RoB) 2.0 assessment [36]. Using the RoB 2.0 assessment six domains of bias were rated for every study, each domain having three rating categories: low RoB, moderate/ some concerns RoB, and high RoB. One reviewer (AK) applied the RoB 2.0 assessment tool to judge the risk of over- or underestimating the effects of the intervention for the outcomes used for the meta-analysis. A second reviewer (ZS) crosschecked the completed RoB 2.0 assessment. Discussion between the reviewers (AK, ZS) resolved disagreement if needed.

The Grades of Recommendation, Assessment, Development and Evaluation (GRADE) was used to rate the overall quality of the evidence and the strength of the recommendations [37]. In accordance with the GRADE Working Group recommendations, the evidence was classified on four levels of quality: very low, low, moderate, and high quality. Moreover, publication bias was determined by computing a funnel plot.

Table 1 Inclusion criteria based on PICOS-parameters

\begin{tabular}{ll}
\hline PICOS-Parameters & Inclusion criteria \\
\hline Population: & Patients after a stroke (ischemic, hemorrhagic) \\
Intervention: & Brain-Computer Interface, Brain-Machine Interface \\
Compare: & Usual/conventional therapies \\
Outcome: & Assessments quantify motor function recovery in upper/lower extremities or/and brain function recovery \\
Study design: & Controlled trials, randomised/randomized controlled trials \\
\hline
\end{tabular}




\section{Data analysis}

For all outcomes representing continuous data, means and standard deviations, sample size, and given $p$-values were entered into the Comprehensive Meta-Analysis Version 3.0 software (Biostat, Englewood, NJ, USA).

\section{Primary outcomes}

The weighted standardized mean differences (SMD) and their corresponding 95\% confidence intervals (95\%CI) were calculated for individual studies and visualised in forest plots separately for upper extremity, lower extremity, and brain function recovery. The analysis included the main outcome measure for motor function recovery or brain function recovery, as specified by the study investigator. The analysis for brain function recovery included the outcome measures such as indices of concentration plus changes in activation and connectivity in the brain function network. Different indices were used to analyse these responses of brain activity. However, if the study investigator did not specify the main outcome, the measure was specified by the first author (AK). Prior to the research, it was decided to metaanalyse the results of the individual studies using the random-effects model with the inversed-variance method due to expected heterogeneity between studies. To test the hypothesis of no among-studies heterogeneity the Q-test with its corresponding degrees of freedom (df) and $p$-value for an alpha level of $5 \%$ was used. Higgins' $\mathrm{I}^{2}$ statistic was used as a measure of heterogeneity, indicating how much of the total observed variance can be explained by the true between studies variation [38]. $\mathrm{T}^{2}$ was used to measure the actual dispersion of variance [39]. In addition, the 95\% prediction interval (95\%CI) was calculated for the overall weighted mean estimate [39]. This indicator shows, in 95\% of cases, that the true effect size in a new study will be within the range of the dispersion of the effect size [39]. The overall mean effect size was expressed in the original metric of the FMA scale. According to the Cochrane handbook in re-expressing SMDs using a familiar instrument, the standard deviation could be obtained as the pooled standard deviation of baseline scores [40].

\section{Secondary outcomes}

To evaluate effect size differences subgroup analyses were performed to compare the treatment effect for: (1) patients in the subacute ( $<6$ months after onset of stroke) and chronic (>6 months after onset of stroke) period after stroke [39] (2) training intensity (high: 5 times per week vs. moderate: 2-3 times per week), (3) training duration (short: 2-3 weeks vs. long: 4-8 weeks, and (4) the follow-up period after training (short-term: 12 weeks vs. long-term: 24-36 weeks). Furthermore, a sensitivity analysis for RoB results was performed.
Studies with overall bias 'high' in the RoB 2.0 Assessments were removed from the meta-analysis to determine the robustness of the pooled effect size [36]. To evaluate the impact of covariates in relation to the effect size, a meta-regression was performed using the random-effects model [39]. Level of impairment at baseline (FMA upper extremity), training duration in weeks (long, short), and the interaction between these two single covariates were chosen as moderators.

\section{Results}

\section{Study selection}

In July 2018, the initial search was performed in the specified databases with the defined search strategy for the identification of studies. The identified 991 studies were imported into the reference management software Endnote (Clarivate Analytics, Philadelphia, USA). After reference import, 326 duplicates were removed, and 665 studies remained. No new references were identified by the search update in March 2019. Figure 1 illustrates the selection process.

Two reviewers (AK, ZS) independently examined whether the relevant studies fitted the population, intervention, comparison, outcome and study design (PICOS) strategy of our research question. After screening titles and abstracts of all studies, full texts of the remaining 61 studies were consulted. Eleven authors were contacted for missing data. Disagreement of selected full texts was resolved with mutual consent. Finally, fourteen studies could be included. The kappa statistic after full text screening was 0.82 . The reviewers (AK, ZS) could not agree on three studies and therefore a third reviewer (CS) was consulted to decide on the studies ' eligibility. The decision was to exclude the three studies. Table 2 provides an overview on all characteristics of the included studies.

\section{Characteristics of studies}

Included studies were published between 2010 and 2017 including small sample sizes ranging between five to 55 patients (mean age between 40.9 and 64.1 years) in the subacute or chronic phase after a stroke in the experimental groups. The BCI training lasted for a minimum of 3 days to maximal 6 weeks with two to three training sessions per week. The Fugl-Meyer Assessment (FMA) for upper extremity was the most used assessment in ten studies, though three studies used a modified upper limb FMA with a maximal score of 54 points (not modified FMA max. Score $=226$ ).

\section{Brain recovery indices}

Four brain recovery indices were described. However not all indices could be included in the meta-analysis due to missing data: 


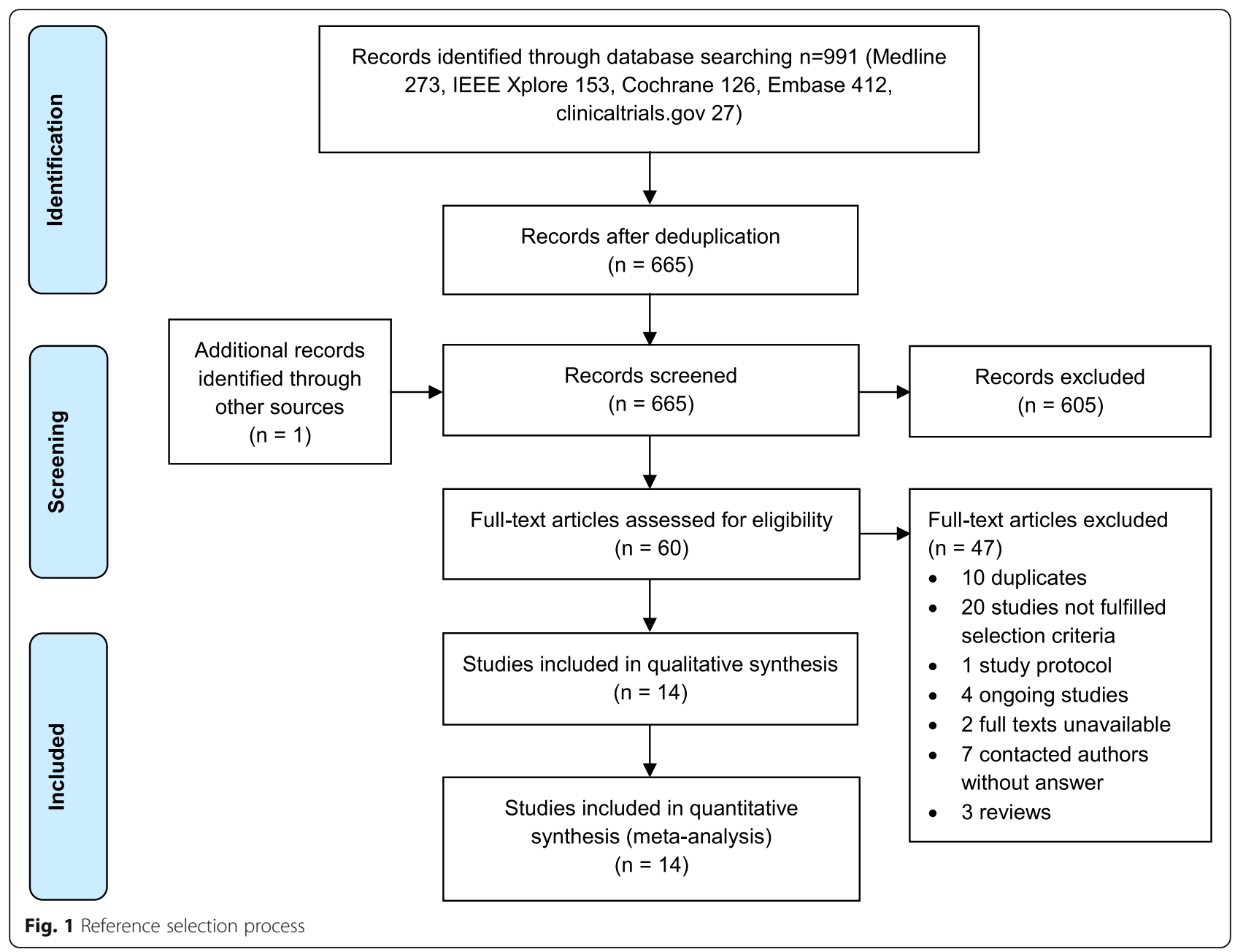

(1) Pichiorri et al. [26] and Ramos-Murguialday et al. [27] used the laterality index measured by fMRI to assess cerebral cortical lateralization to quantify brain recovery. In accordance to Pichiorri [26], the lateralization index expresses the normalized difference between the number of active voxels in the ipsilesional and contralesional. The lateralization index yields a value of 1 or -1 when the activity was purely contralesional or ipsilesional respectively. Only patients with subcortical lesions were considered for a lateralization index assessment.

(2) Várkuti et al. [28] and Biasiucci et al. [19] associated motor function recovery in patients in the chronic phase after stroke with quantitative indicators of functional neuroplasticity. The authors measured changes in functional connectivity after $\mathrm{BCI}$ by comparing the resting state fMRI pre- and post $\mathrm{BCI}$ training. According to the authors, the functional connectivity changes might reflect re-organisational processes that have occurred between pre- and post BCI training [28].
(3) Chung et al. $[14,20]$ examined the effect of BCI based on functional electrical stimulation (FES) on brain activation in patients in the chronic phase after stroke. EEG brainwave patterns were calculated as the ratio of sensorimotor (SMR) and Mid-Beta waves to Theta waves ((SMR+ Mid-Beta)/ Theta). The authors demonstrated significant effects for the EEG brainwave patterns in the frontopolar regions attention index 1 (Fp1) and 2 (Fp2), and frontopolar 1 (Fp1) [20].

(4) A fourth index was mentioned by Ang et al. [14]. The authors collected EEG data during the BCI-Manus therapy to detect interhemispheric asymmetry using the brain symmetry index that can range between zero (lowest level of asymmetry) and one (highest level of asymmetry) [41]. The averaged brain symmetry index from all 12 sessions for the eleven participants in the BCIT group was analysed. Ang et al. and Anastasi et al. proposed that the brain symmetry index can be used as a prognostic measure for BCIbased stroke rehabilitation $[14,42]$. However, the 


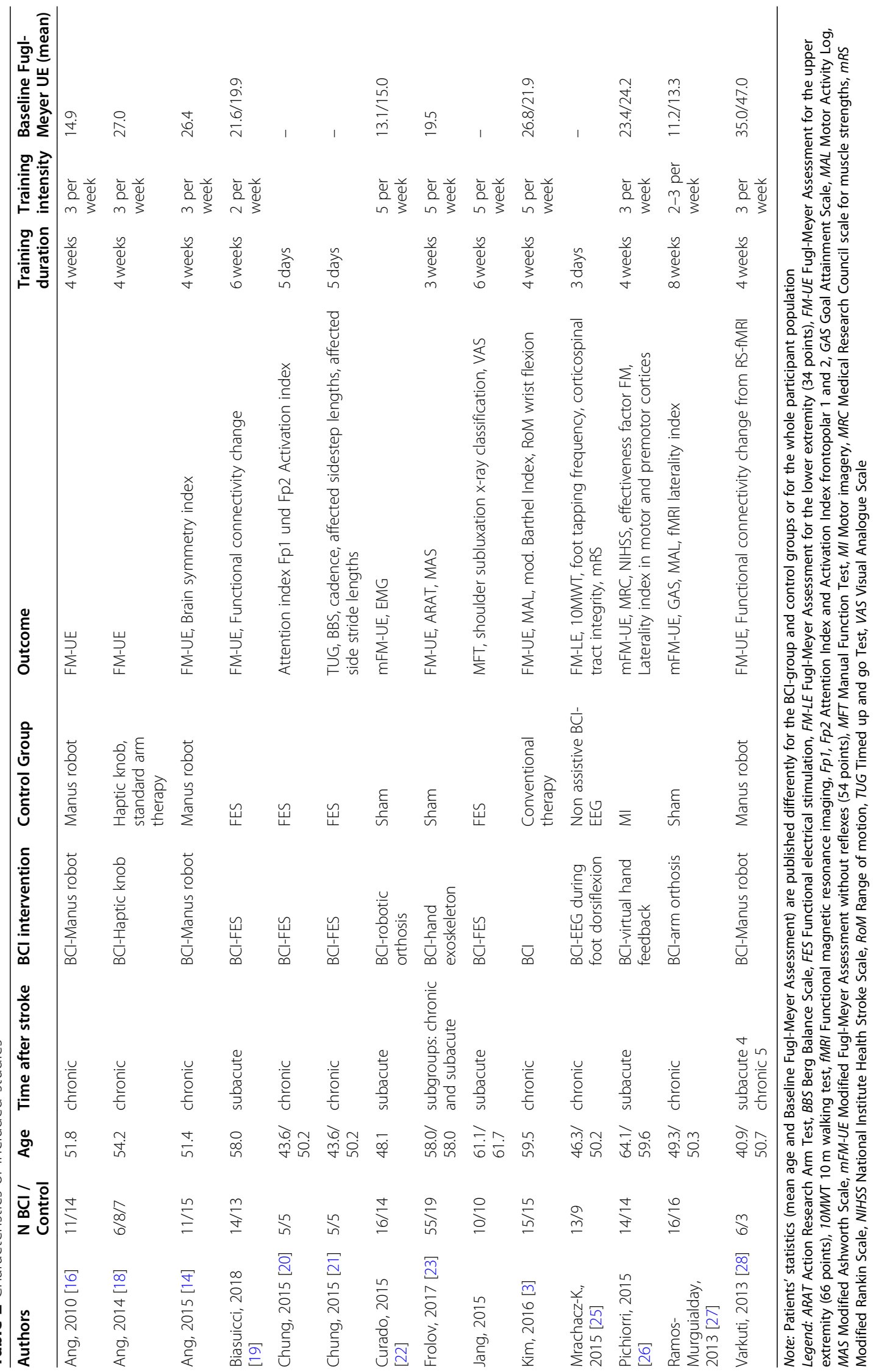


study from Ang et al. could not be included in the meta-analysis due to missing data from the control group.

\section{Risk of bias within studies}

Figures 2 and 3 show the results of the RoB evaluation [36]. Qualitative assessment showed low risk in measurement of outcomes, missing outcome data, and selection of the reported results. Moreover, studies showed some concerns in the randomisation process and deviation from intended intervention. Only two studies had a high risk of overall bias.

\section{Risk of bias across studies}

RoB results for all studies for the upper extremity were pooled and presented in funnel plots (Additional file 3). Looking for missing studies right of the mean, in accordance to the developed method, the CMA software adjusted two studies. Using the Trim and Fill method, the imputed point estimate was 0.43 (95\%CI: 0.22 to 0.64 ). This indicates a slightly higher effect size, but still very similar to the point estimate of the pooled studies. The adjusted value was equivalent to the observed value and the weighted effect size was 0.39 ( $95 \% \mathrm{CI}: 0.17$ to 0.62 ). It can be concluded, if adjusting the effect to remove the possible bias or asymmetry, the resulting effect would remain unchanged. However, included studies did not indicate a significant asymmetry suggesting a low publication bias.

\section{GRADE- evidence profile table}

After the evidence was summarised, small sample sizes, the width and overlap of confidence intervals, heterogeneity and generalisability were taken into consideration. One reviewer (AK) created a GRADE evidence profile table (Table 3) to present key information on five defined outcomes (GRADEproGDT, 2015). A second reviewer (ZS) crosschecked the results. The reviewers (AK, ZS) resolved disagreement by discussion. This was the case in the rating of the outcome 'brain function recovery' for 'not serious' or 'serious'. The final decision was 'serious'.

\section{Primary outcomes- effect of BCIT on motor function recovery}

Eleven studies [3, 14, 16, 18, 19, 22-24, 26-28] with (329 patients in total) were included in a meta-analysis evaluating the effect of BCIT versus conventional therapy alone on motor function recovery of the upper extremity in patients after a stroke (Fig. 4). The weighted SMD was 0.39 (95\% CI: 0.17 to 0.62 ) with a $95 \% \mathrm{PI}$ ranging from 0.13 to $0.66(\mathrm{Z}=$ $3.45, p=0.001)$. Heterogeneity was very low $\left(\mathrm{I}^{2}=0.00 ; \mathrm{Q}=\right.$ $3.53, \mathrm{df}=11, p=0.982$ ). In this set of 11 studies the variance of the distribution of the effect sizes was $\mathrm{T}^{2}=0.00$.

Two studies [21, 25] were included in a meta-analysis for the effect of BCIT versus conventional therapy alone on motor function recovery of the lower extremity in patients after a stroke with 32 patients (Fig. 5). The weighted SMD was $0.41(95 \% \mathrm{CI} ;-0.29$ to $1.12 ; \mathrm{Z}=1.15 ; p=0.252)$. Heterogeneity was very low $\left(\mathrm{I}^{2}=0.00 ; \mathrm{Q}=0.02 ; \mathrm{df}=1\right.$, $p=0.880$ ). The variance of the distribution of the effect sizes in this sample of the two studies was $\mathrm{T}^{2}=0.00$.

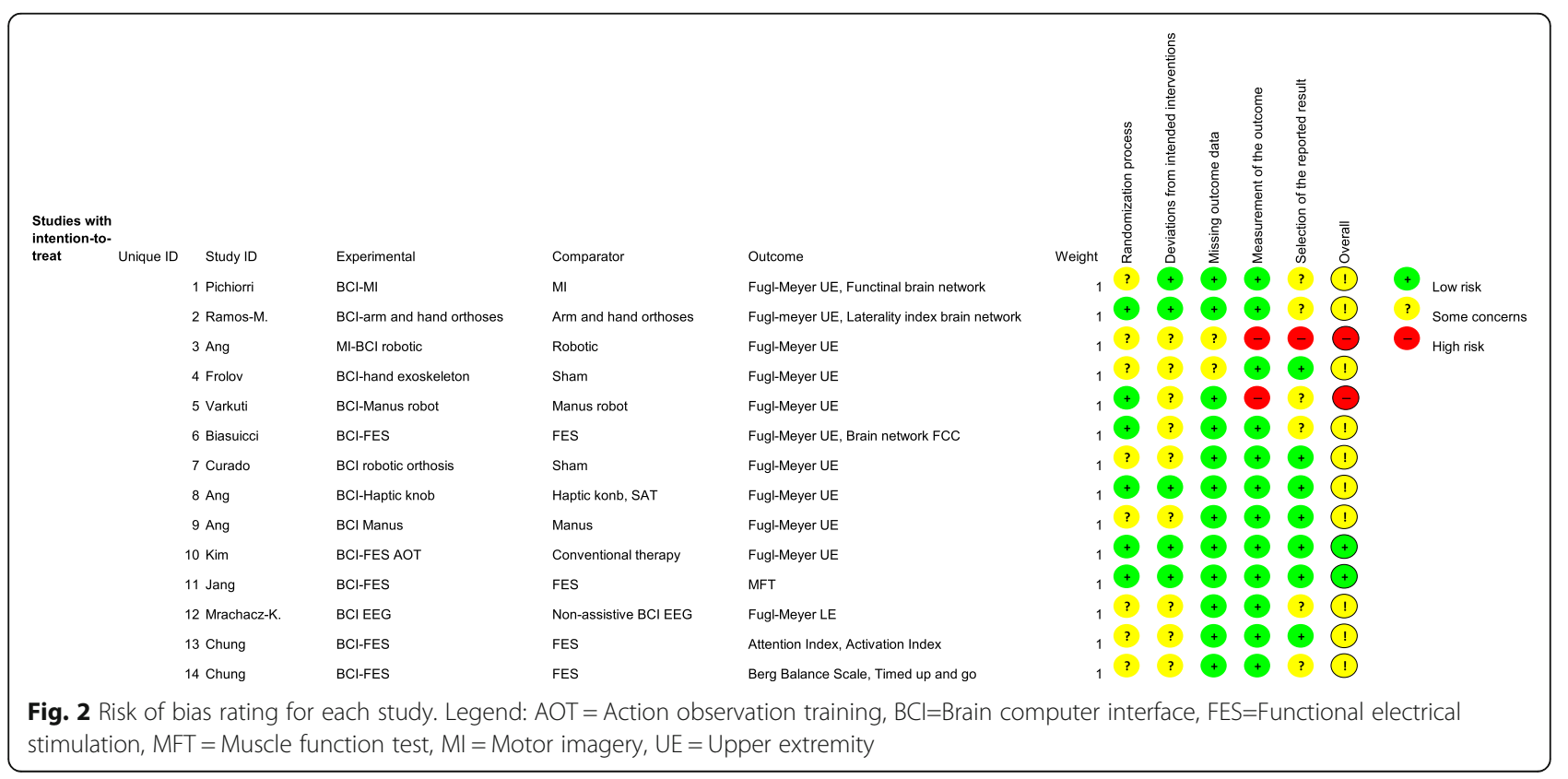




\section{As percentage (intention-to-treat)}

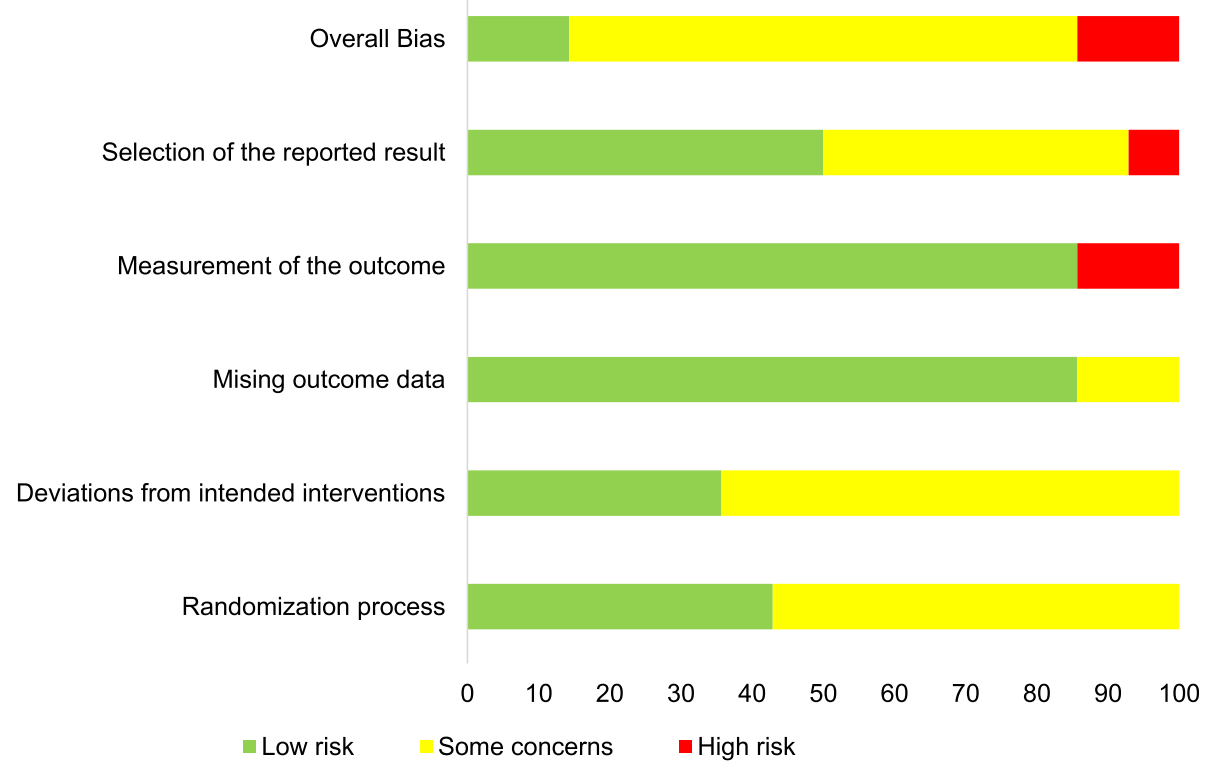

Fig. 3 Domains rated as percentage of all studies

The meta-analysis, examining the effect of BCIT versus conventional therapy alone on brain function recovery in patients after a stroke, included five studies with 85 patients (Fig. 6) [19, 20, 26-28]. The overall weighted SMD was 1.11 (95\% CI; 0.64 to 1.59$)$ with a 95\%PI ranging from 0.33 to $1.89(\mathrm{Z}=4.82 ; p=0.000)$. Heterogeneity was very low $\left(\mathrm{I}^{2}=0.00 ; \mathrm{Q}=3.12\right.$, $\left.\mathrm{df}=5, p=0.000\right)$. The variance of the distribution of the effect sizes in this sample of five studies was $\mathrm{T}^{2}=0.00$.

\section{Secondary outcomes}

Different subgroups were defined to compare treatment effects and their influence on the effect size: different kinds of the BCIT, time since stroke onset, training intensity and duration, and follow-up period. The subgroup and sensitivity analyses regarding upper extremity motor function were performed and were presented in Additional files 4 and 5 .

\section{Subgroup analysis: time since stroke onset and training intensity}

There is a statistically significant effect for both groups regarding time after stroke: subacute patients had a value of 0.57 (95\%CI: 0.19 to $0.96 ; \mathrm{Z}=2.90 ; p=0.004$ ) and chronic patients a value of 0.39 (95\%CI: 0.09 to $0.68 ; \mathrm{Z}=2.59 ; p=0.010)$. Within the 'moderate intensity' subgroup, the weighted effect size was also in favour of the BCIT group as compared to the control group. However, the result was statistically not significant ( $p=$ 0.095). Within the 'high intensity' subgroup, the weighted effect size was 0.58 (95\%CI: 0.24 to $0.92 ; \mathrm{Z}=$ 3.33; $p=0.001$ ) and statistically significant.

The weighted effect size for patients in the subacute or chronic phase after stroke can be interpreted as a small to medium effect.

Subgroup analysis: training duration and follow up period Using the random-effects model, the weighted overall effect sizes were statistically significant for: (1) short duration training (two to 3 weeks) with a value of 0.54 (95\%CI: 0.19 to $0.89 ; \mathrm{Z}=3.06 ; p=0.002$ ), (2) long duration training (four to 8 weeks) with a value of 0.31 (95\%CI: 0.06 to $0.56 ; \mathrm{Z}=2.44 ; p=0.016$ ), and (3) longterm follow-up ( 24 to 36 weeks) with a value of 0.56 . (95\%CI: 0.01 to $1.11 ; Z=1.99 ; p=0.047$ ).

The weighted effect size for short and long duration training, and long-term follow-up can be interpreted as a small to medium effect.

\section{Sensitivity analysis}

For the sensitivity analysis two studies [14, 27] were removed after being rated high-risk in the domain 'overall bias' $(\mathrm{SMD}=0.42 ; 95 \% \mathrm{CI}$ : 0.18 to $0.66 ; \mathrm{Z}=3.46 ; p=$ $0.001)$. The heterogeneity was very low $\left(\mathrm{I}^{2}=0.00\right)$ and statistically not significant $(\mathrm{Q}=2.97, \mathrm{df}=9, p=0.965)$.

\section{Meta-regression}

A meta-regression was performed to assess the effect three covariates on the effect size: training duration, level of impairment, and the interaction between training 


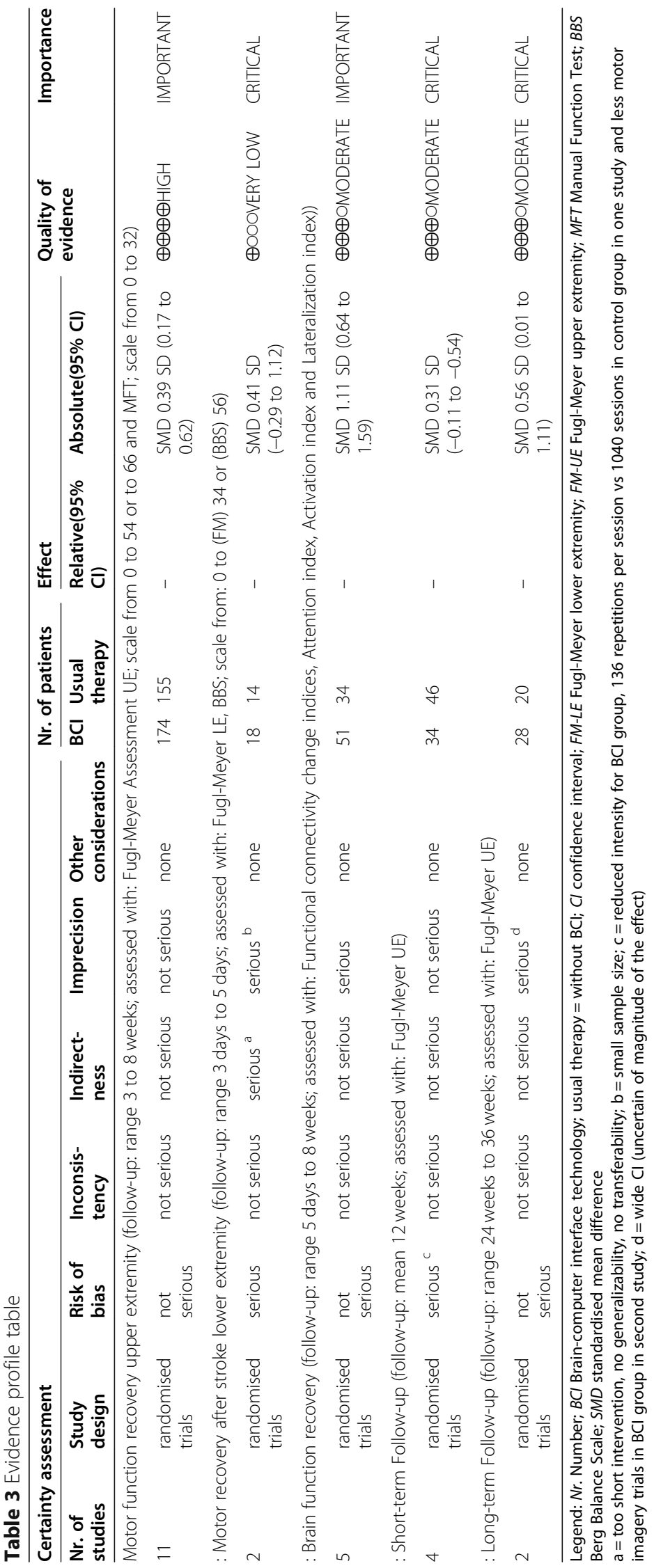


Effect of $\mathrm{BCl}$ added to conventional therapy versus control intervention on recovery of upper extremity

\begin{tabular}{|c|c|c|c|c|c|c|c|c|c|}
\hline \multirow[t]{2}{*}{$\underline{\text { Study name }}$} & \multirow[t]{2}{*}{ Subgroup } & \multicolumn{4}{|c|}{ Statistics for each study } & \multicolumn{2}{|c|}{$\underline{\text { Sample size }}$} & \multirow{2}{*}{\multicolumn{2}{|c|}{$\begin{array}{l}\text { Std diff in means } \\
\text { and } 95 \% \mathrm{Cl}\end{array}$}} \\
\hline & & $\begin{array}{l}\text { Std diff } \\
\text { in means }\end{array}$ & $\begin{array}{l}\text { Lower } \\
\text { limit }\end{array}$ & $\begin{array}{l}\text { Upper } \\
\text { limit }\end{array}$ & o-Value & $\begin{array}{l}\mathrm{BCl} \\
\text { grouph }\end{array}$ & $\begin{array}{l}\text { Control } \\
\text { ithout } \mathrm{BCl}\end{array}$ & & \\
\hline Ang 2010 & - & 0,27 & $-0,52$ & 1,07 & 0,499 & 11 & 14 & & \\
\hline Ang 2014 & Robot & 0,03 & $-1,30$ & 1,35 & 0,969 & 3 & 8 & & \\
\hline Ang 2014 & SAT & 0,62 & $-0,76$ & 2,00 & 0,381 & 3 & 7 & & \\
\hline Ang 2015 & - & 0,10 & $-0,69$ & 0,89 & 0,809 & 11 & 14 & & \\
\hline Biasiucci 2018 & - & 0,34 & $-0,42$ & 1,10 & 0,378 & 14 & 13 & & \\
\hline Curado 2015 & - & 0,52 & $-0,18$ & 1,23 & 0,144 & 16 & 16 & & \\
\hline Frolov 2017 & - & 0,70 & 0,17 & 1,24 & 0,010 & 55 & 19 & 7 & \\
\hline Jang 2015 & - & 0,38 & $-0,50$ & 1,26 & 0,399 & 10 & 10 & & \\
\hline Kim 2016 & - & 0,52 & $-0,20$ & 1,25 & 0,158 & 15 & 15 & & \\
\hline Pichiorri 2014 & - & 0,34 & $-0,41$ & 1,09 & 0,370 & 14 & 14 & - & \\
\hline Ramos-M. 2013 & & 0,22 & $-0,47$ & 0,92 & 0,530 & 16 & 16 & - & \\
\hline \multirow[t]{4}{*}{ Varkuti 2012} & - & 0,05 & $-0,98$ & 1,09 & 0,920 & 6 & 9 & & \\
\hline & & 0,39 & 0,17 & 0,62 & 0,001 & & & 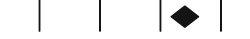 & | \\
\hline & & & & & & & & $\begin{array}{llll}-2,00 & -1,00 & 0,00 & 1,00\end{array}$ & 2,00 \\
\hline & & & & & & & & Contrc & \\
\hline
\end{tabular}

Fig. 4 Effect of brain computer interface training added to conventional therapy versus control intervention on upper extremity motor function. Legend: $\mathrm{BCl}=$ Brain computer interface, $\mathrm{Cl}=$ Confidence interval, SAT = Standard arm therapy, $\mathrm{Std}=$ Standard deviation

duration and FMA baseline value. However, none of the three covariates showed a significant influence (see Additional file 6).

\section{Discussion}

In this systematic review, 14 studies with patients after a stroke were included to investigate the effect of BCI on recovery after stroke. All studies focussed on the effect of BCIT added to conventional therapy on motor and brain function recovery in patients after a stroke ischemic or hemorrhagic stroke. Not all studies reported information regarding lesion location. However, if reported, the stroke was located in cortical and subcortical areas. Furthermore, studies involved more patients in the chronic phase after stroke than in the subacute phase after stroke. Most studies presented a low RoB in the outcome measurements, in the deviation from the intended intervention, and in the randomisation process. Studies focussing on motor function recovery for upper extremity were rated as 'high quality' and focussing on brain function recovery were rated as 'moderate certainty'. Both study groups presented low sample sizes, wide CIs, and very short intervention periods. None of the included studies reported adverse events.

Meta-analyses indicated a statistically significant benefit of BCIT on motor function recovery of the upper extremity and brain function recovery in patients after a stroke. For the upper extremity, a statistically significant benefit could be interpreted as 5.4 to 8.1 points on the FMA of the upper extremity [3, 22, 23]. Furthermore, the analyses showed homogeneity among studies with the most participants and methodological sound experimental protocols.
Only two trials focussed on motor function recovery of the lower extremity. However, both studies presented low sample sizes, large confidence intervals, and poor methodological quality making it impossible to draw clear-cut conclusions [21, 25].

Regarding the upper extremity, in a sensitivity analysis, two studies rated as 'high risk' in the RoB were excluded. The exclusion had a small effect on the overall weighted estimate indicating its robustness against RoB in the individual studies.

The subgroup analyses demonstrated significant effects for subacute and chronic patients. The weighted effect size for subacute patients had a large effect size of 0.57 compared to the small to medium effect size of 0.39 for chronic patients. Furthermore, for the high intensity training a large effect size of 0.58 was found whereas both the weighted effect size for short and long duration training was statistically significant. However, due to the trend to reach a recovery plateau in patients in the chronic phase after stroke, a high intensity BCIT (two to three times a week) with a training duration between three to 6 weeks might be an efficient approach in clinical practice.

To evaluate the impact of the covariates training duration, level of impairment, and the interaction between training duration and FMA baseline value on the effect size, a meta-regression was performed using the random-effects model [36]. However, no significant effects on the overall pooled estimate were detected. It remains to be investigated whether the training duration combined with the training intensity could have a significant influence on the effect size. 


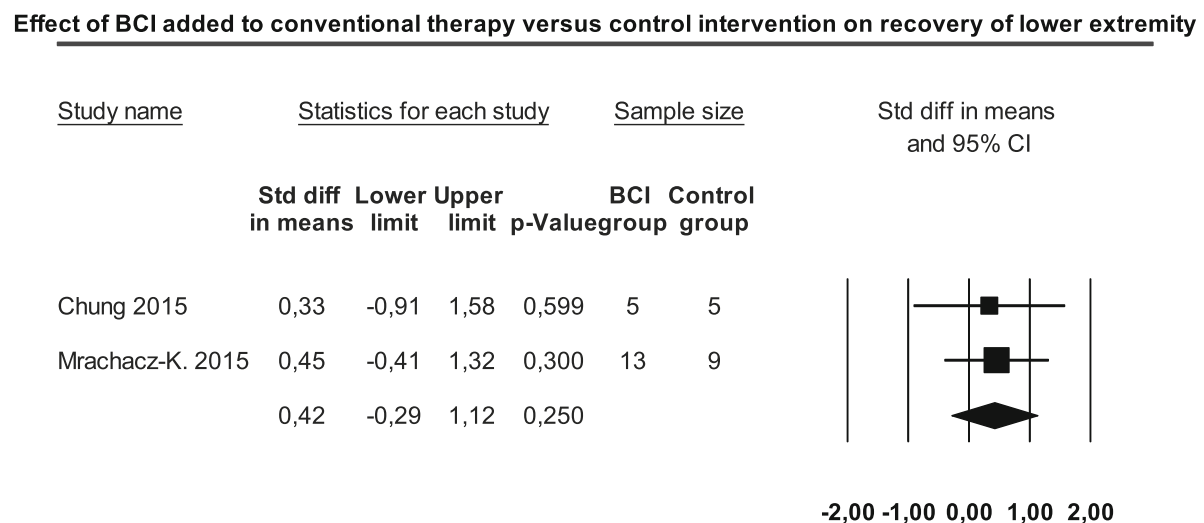

Control $\mathrm{BCl}$

Fig. 5 Effect of brain computer interface training added to conventional therapy versus control intervention on lower extremity function. Legend: $\mathrm{BCl}=$ Brain computer interface, $\mathrm{Cl}=$ Confidence interval, Std $=$ Standard deviation

None of the 14 included studies used standardised assessments to evaluate MI ability. Malouin et al., described a mental slowing after stroke leading to more difficulties to generate a mental representation of movement $[43,44]$. To determine participant benefits of BCIT based on MI, it is important to evaluate his/her MI ability [45]. Different valid and reliable assessments to evaluate patients MI ability are available such as the Kinaesthetic and Visual Imagery Questionnaire [46].

Furthermore, there were no standards for introduction or training of MI reported and there might be differences in the MI methods applied among facilities. A detailed description would be helpful to transfer the successfully implemented MI strategies into clinical routine. We further speculate that the potential of BCIT might be even higher if patients would receive a systematic MI introduction and training $[47,48]$.

A correlation between brain function recovery and motor function recovery was mentioned by RamosMurguialday et al. [27]. Authors mentioned a correlation between the laterality index changes and FMA subscale hand score $(r=0.54, p=0.05)$. Pichiorri et al. examined,

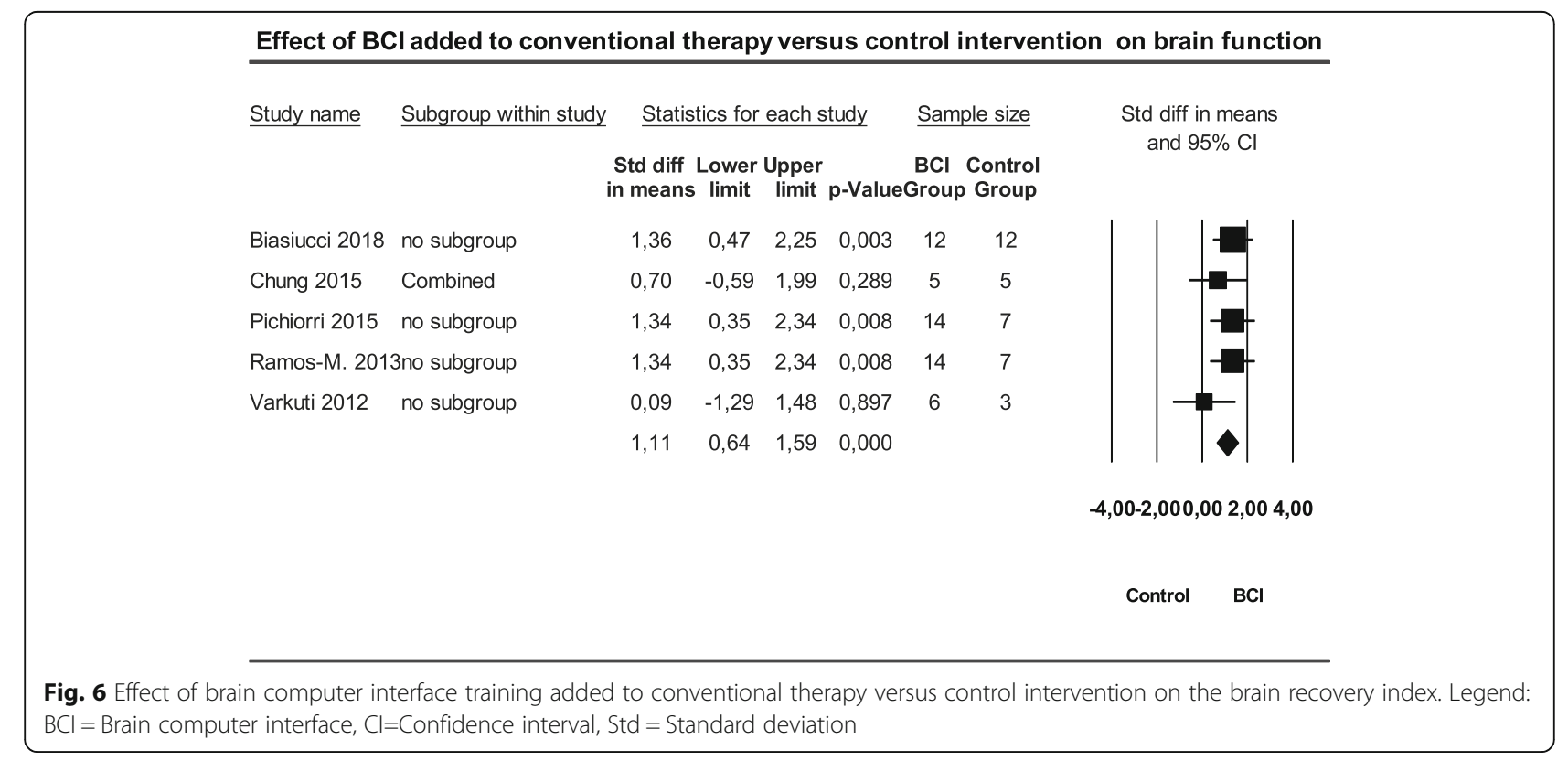


whether the possible changes in the intra-hemispheric networks correlated with the FMA score [26]. Their analysis detected a positive correlation between the increase in the laterality index value and the scoring in the FMA scale in the BCI group in the beta 1 bands $(r=0.57, p=$ $0.034)$, beta $2(r=0.60, p=0.024)$, and gamma bands $(r=0.61, p=0.023)$. The same laterality index was not significant for the unaffected hemisphere.

Moreover, Ang et al. [14] reported a correlation $(r=-0.62$, $p=0.044)$ between the brain symmetry index, indicating a trend towards less asymmetry values and an improved FMA scoring.

Correlations between brain function recovery and cognitive assessments were not reported in the literature. However, the evaluation of cognitive capacities in patients with severe motor disabilities might have relevant implications for the BCIT systems [49]. Current BCIT systems are not well suited for use outside the clinic or research laboratory due to their large size, high costs, and lengthy set up time. Furthermore, the BCIT systems require highly trained personnel. However, future investigations should involve controlled experiments using low-cost BCIT systems. A section 'state of the art' of studies about costs and additional information about studies is provided in the supplementary material.

\section{Limitations and strengths}

The systematic review process might have been confounded by some factors. The search was only performed in English language databases that published abstracts in English. However, the main literature sources are English-speaking peer-reviewed journals and conferences. No abstract or full text were excluded because it was published in a different language than English. The calculated effect sizes ignored the fact that conventional therapy compared with BCIT varied among the 14 included studies. However, the composition of the search strategy and the search itself were conducted by a professional research librarian from the University of Zurich in accordance with the review protocol providing a comprehensive search and detailed knowledge of different databases with a medical or technical focus.

\section{Conclusion}

Based on the results of the present review, we recommend a BCIT combined with conventional therapy for a duration of 4 weeks or longer, with a preference for high intensity training of five times per week. BCIT combined with a wide range of different interventions reflects complexity and variety in of its applicability. We further recommend implementing assessments of neuropsychological parameters such as attention, concentration or cognition as well as MI ability measures, such as the KVIQ, to evaluate patients' capabilities important for the mental training. An important topic for future work will be the examination of motor function recovery of the lower extremities in patients after a stroke. Furthermore, there is abundant scope for further progress in determining different aspects of the effect of $\mathrm{BCIT}$ on brain recovery in addition to functional connectivity. To develop a full picture of clinical factors that influence the effect size in motor recovery of upper extremities of patients after stroke, additional studies are needed to analyse parameters, such as patients' age, lesion location, performance of MI ability, affected hemisphere, or type of lesion. Moreover, future studies should include long-term follow-ups 24-36 weeks after intervention begin in patients after a stroke. The interaction between baseline Fugl-Meyer Assessment score and training duration showed a nosignificant effect. However, the interaction between the covariates training duration and training intensity should be further investigated.

\section{Supplementary information}

Supplementary information accompanies this paper at https://doi.org/10. 1186/s12883-020-01960-5.

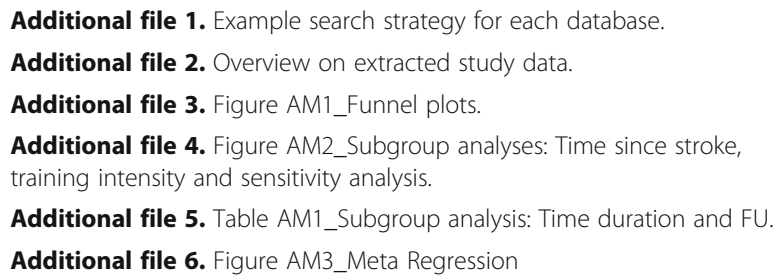

Additional file 5. Table AM1_Subgroup analysis: Time duration and FU. Additional file 6. Figure AM3_Meta Regression

\section{Abbreviations}

BCl: Brain-computer interface; SMD: Standardized mean difference; Cl: Confidence interval; PI: Prediction interval; EEG: Electroencephalography; MEG: Magnetoencephalography; fNIRS: Functional near-infrared spectroscopy; fMRI: Functional magnetic resonance imaging; ECoG: Electrocorticography; LEPS: Local field potentials; EFPs: Epidural field potentials; MI: Motor imagery; RoB: Risk of bias; GRADE: Grades of recommendation, assessment, development and evaluation; df: Degrees of freedom; FMA: Fugl-meyer assessment; KVIQ: The kinaesthetic and visual imagery questionnaire

\section{Acknowledgements}

We are grateful to Dr. Sabine Klein (SK) from the University of Zurich for her support with the literature search. We would like to thank the authors for their answers and Jose Millan for managing to provide the missing values, and Prof. S. J. Hawkins from the University of Southampton for proofreading.

\section{Authors' contributions}

All authors contributed to the study conception and design. The literature search, selection and evaluation procedures were carried out by AK, ZS, and CSA. Data extraction, data control, and classification/evaluation of the literature were performed by AK and ZS. AK, JT, and CSA carried out data analysis and interpretation. AK drafted the manuscript and was critically reviewed and revised by ZS, JT, and CSA. All authors gave permission to the submission of the manuscript and agree to be accountable for all aspects of the work in ensuring that questions related to the accuracy or integrity of any part of the work are appropriately investigated and resolved. The author (s) read and approved the final manuscript.

\section{Funding}

The project was not funded. The literature search and retrieval were supported by the library of the University of Zurich in cooperation with the rehabilitation centre Reha Rheinfelden. 


\section{Availability of data and materials}

All data presented in this systematic review are derived from published studies and are available from the first author on reasonable request.

\section{Ethics approval and consent to participate}

Not applicable. For the systematic review we analysed already published literature only.

\section{Consent for publication}

Not applicable - Systematic literature review.

\section{Competing interests}

None declared.

\section{Author details}

'Department of Health Professions, Bern University Applied Science, Schwarztorstrasse 48, 3007 Bern, Switzerland. Private Practice, Baslerstrasse 60, 4102 Binningen, Switzerland. ${ }^{3}$ Research Department, Reha Rheinfelden, Salinenstrasse 98, 4310 Rheinfelden, Switzerland. ${ }^{4}$ Vrije Universiteit Brussel, Pleinlaan 2, 1050 Brussels, Belgium. ${ }^{5}$ Department of Engineering and Information Technology, Pestalozzistrasse 20, 3401 Burgdorf, Switzerland. ${ }^{6}$ Department of Sport, Exercise and Health, University of Basel, Birsstrasse 320 B, 4052 Basel, Switzerland.

Received: 21 April 2020 Accepted: 14 October 2020

Published online: 22 October 2020

\section{References}

1. Feigin $\mathrm{VL}$, Forouzanfar MH, Krishnamurthi $\mathrm{R}$, Mensah GA, Connor M, Bennett DA, Moran AE, Sacco RL, Anderson L, Truelsen T, et al. Global and regional burden of stroke during 1990-2010: findings from the global burden of disease study 2010. Lancet. 2014;383(9913):245-54

2. Mackay-Lyons MJ, Makrides L. Longitudinal changes in exercise capacity after stroke. Arch Phys Med Rehabil. 2004;85(10):1608-12

3. Kim T, Kim S, Lee B. Effects of action observational training plus braincomputer Interface-based functional electrical stimulation on paretic arm motor recovery in patient with stroke: a randomized controlled trial. Occup Ther Int. 2016;23(1):39-47.

4. Wolpaw JR, Birbaumer N, McFarland DJ, Pfurtscheller G, Vaughan TM. Braincomputer interfaces for communication and control. Clin Neurophysiol. 2002;113(6):767-91.

5. Guger C, Allison B, Lebedev M. Brain-computer interface research : a stateof-the-art summary 6. In: Springer Briefs in electrical and computer engineering. Cham, Switzerland: Springer; 2017. 1 online resource.

6. Dickstein R, Deutsch JE: Motor imagery in physical therapist practice. Phys Ther. 2007:87(7):942-53. https://doi.org/10.2522/ptj.20060331. Epub 2007 May 1.

7. Decety J. The neurophysiological basis of motor imagery. Behav Brain Res. 1996;77(1-2):45-52

8. Solodkin A, Hlustik P, Chen EE, Small SL. Fine modulation in network activation during motor execution and motor imagery. Cereb Cortex. 2004; 14(11):1246-55.

9. Braun S, Kleynen M, van Heel T, Kruithof N, Wade D, Beurskens A. The effect of mental practice in neurlogical rehabilitation: a systematic review and meta-analysis. Front Hum Neurosci. 2013;7:390.

10. Guerra ZF, Lucchetti ALG, Lucchetti G. Motor imagery training after stroke: a systematic review and meta-analysis of randomized controlled trials. J Neurol Phys Ther. 2017:41(4):205-14.

11. Monge-Pereira E, Molina-Rueda F, Rivas-Montero FM, Ibanez J, Serrano الر, Alguacil-Diego IM, Miangolarra-Page JC. Electroencephalography as a poststroke assessment method: an updated review. Neurologia. 2017;32(1):40-9.

12. Carvalho R, Dias N, Cerqueira JJ. Brain-machine interface of upper limb recovery in stroke patients rehabilitation: a systematic review. Physiother Res Int. 2019;24(2):e1764

13. Cervera MA, Soekadar SR, Ushiba J, Millan JDR, Liu M, Birbaumer N, Garipelli G. Brain-computer interfaces for post-stroke motor rehabilitation: a metaanalysis. Ann Clin Transl Neurol. 2018;5(5):651-63.

14. Ang KK, Chua KS, Phua KS, Wang C, Chin ZY, Kuah CW, Low W, Guan C. A randomized controlled trial of EEG-based motor imagery brain-computer interface robotic rehabilitation for stroke. Clin EEG Neurosci. 2015;46(4):310-20.
15. Ang KK, Guan C. EEG-based strategies to detect motor imagery for contro and rehabilitation. IEEE Trans Neural Syst Rehabil Eng. 2017:25(4):392-401.

16. Ang KK, Guan C, Chua KS, Ang BT, Kuah C, Wang C, Phua KS, Chin ZY, Zhang $\mathrm{H}$. Clinical study of neurorehabilitation in stroke using EEG-based motor imagery brain-computer interface with robotic feedback. Conf Proc IEEE Eng Med Biol Soc. 2010;2010:5549-52.

17. Ang KK, Guan C, Phua KS, Wang C, Teh I, Chen CW, Chew E. Transcranial direct current stimulation and EEG-based motor imagery $\mathrm{BCI}$ for upper limb stroke rehabilitation. Conf Proc IEEE Eng Med Biol Soc. 2012;2012:4128-31.

18. Ang KK, Guan C, Phua KS, Wang C, Zhou L, Tang KY, Ephraim Joseph GJ, Kuah CW, Chua KS. Brain-computer interface-based robotic end effector system for wrist and hand rehabilitation: results of a three-armed randomized controlled trial for chronic stroke. Front Neuroeng. 2014;7:30.

19. Biasiucci A, Leeb R, Iturrate I, Perdikis S, Al-Khodairy A, Corbet T, Schnider A, Schmidlin $T$, Zhang $H$, Bassolino $M$, et al. Brain-actuated functional electrical stimulation elicits lasting arm motor recovery after stroke. Nat Commun. 2018;9(1):2421.

20. Chung E, Kim JH, Park DS, Lee BH. Effects of brain-computer interface-based functional electrical stimulation on brain activation in stroke patients: a pilot randomized controlled trial. J Phys Ther Sci. 2015;27(3):559-62.

21. Chung E, Park SI, Jang YY, Lee BH. Effects of brain-computer interface-based functional electrical stimulation on balance and gait function in patients with stroke: preliminary results. J Phys Ther Sci. 2015;27(2):513-6.

22. Curado MR, Cossio EG, Broetz D, Agostini M, Cho W, Brasil FL, Yilmaz O, Liberati G, Lepski G, Birbaumer N, et al. Residual upper arm motor function primes innervation of paretic forearm muscles in chronic stroke after brainmachine Interface (BMI) training. PLoS One. 2015;10(10):e0140161.

23. Frolov AA, Mokienko O, Lyukmanov R, Biryukova E, Kotov S, Turbina L, Nadareyshvily G, Bushkova Y. Post-stroke rehabilitation training with a motorimagery-based brain-computer Interface (BCI)-controlled hand exoskeleton: a randomized controlled multicenter trial. Front Neurosci. 2017;11:400.

24. Jang $\mathrm{YY}$, Kim TH, Lee BH. Effects of brain-computer Interface-controlled functional electrical stimulation training on shoulder subluxation for patients with stroke: a randomized controlled trial. Occup Ther Int. 2016;23(2):175-85.

25. Mrachacz-Kersting N, Jiang N, Stevenson AJ, Niazi IK, Kostic V, Pavlovic A, Radovanovic S, Djuric-Jovicic M, Agosta F, Dremstrup K, et al. Efficient neuroplasticity induction in chronic stroke patients by an associative braincomputer interface. J Neurophysiol. 2016;115(3):1410-21.

26. Pichiorri F, Morone G, Petti M, Toppi J, Pisotta I, Molinari M, Paolucci S, Inghilleri $M$, Astolfi L, Cincotti F, et al. Brain-computer interface boosts motor imagery practice during stroke recovery. Ann Neurol. 2015;77(5):851-65.

27. Ramos-Murguialday A, Broetz D, Rea M, Laer L, Yilmaz O, Brasil FL, Liberati G, Curado MR, Garcia-Cossio E, Vyziotis A, et al. Brain-machine interface in chronic stroke rehabilitation: a controlled study. Ann Neurol. 2013;74(1):100-8.

28. Varkuti B, Guan C, Pan Y, Phua KS, Ang KK, Kuah CW, Chua K, Ang BT, Birbaumer N, Sitaram R. Resting state changes in functional connectivity correlate with movement recovery for $\mathrm{BCl}$ and robot-assisted upperextremity training after stroke. Neurorehabil Neural Repair. 2013;27(1):53-62.

29. Monge-Pereira E, Ibanez-Pereda J, Alguacil-Diego IM, Serrano JI, SpottornoRubio MP, Molina-Rueda F. Use of electroencephalography brain-computer interface systems as a rehabilitative approach for upper limb function after a stroke: a systematic review. PM R. 2017;9(9):918-32.

30. Orrison WW, Lewine J, Sanders J, Hartshorne MF. Functional brain imaging. St. Louis: Elsevier health sciences; 1995.

31. Rondina JM, Filippone M, Girolami M, Ward NS. Decoding post-stroke motor function from structural brain imaging. Neurolmage Clin. 2016;12:372-80.

32. Schulz R, Braass H, Liuzzi G, Hoerniss V, Lechner P, Gerloff C, Hummel FC. White matter integrity of premotor-motor connections is associated with motor output in chronic stroke patients. Neurolmage Clin. 2015:7:82-6.

33. Shamseer L, Moher D, Clarke M, Ghersi D, Liberati A, Petticrew M, Shekelle P, Stewart LA, Group P-P. Preferred reporting items for systematic review and meta-analysis protocols (PRISMA-P) 2015: elaboration and explanation. BMJ. 2015;350:97647.

34. Moher D, Liberati A, Tetzlaff J, Altman DG, The PG. Preferred reporting items for systematic reviews and meta-analyses: the PRISMA statement. PLoS Med. 2009:6(7):e1000097.

35. Landis JR, Koch GG. The measurement of observer agreement for categorical data. Biometrics. 1977;33(1):159-74.

36. Higgins JP, Altman DG, Gotzsche PC, Juni P, Moher D, Oxman AD, Savovic J, Schulz KF, Weeks L, Sterne JA. The Cochrane Collaboration's tool for assessing risk of bias in randomised trials. BMJ. 2011;343:d5928. 
37. How to GRADE [Internet].figshare; 2018 [cited 2020Apr1] [https://figshare. com/articles/How to GRADE/6818894/1].

38. Higgins JP, Thompson SG. Quantifying heterogeneity in a meta-analysis. Stat Med. 2002:21(11):1539-58.

39. Borenstein M. Introduction to meta-analysis. Chichester: John Wiley \& Sons; 2009.

40. Higgins JPT, Green S (editors). Cochrane Handbook for Systematic Reviews of Interventions Version 5.1.0 [updated March 2011]. The Cochrane Collaboration; 2011. Available from www.handbook.cochrane.org.

41. Xin X, Chang J, Gao Y, Shi Y. Correlation between the revised brain symmetry index, an EEG feature index, and short-term prognosis in acute ischemic stroke. J Clin Neurophysiol. 2017;34(2):162-7.

42. Agius Anastasi A, Falzon O, Camilleri K, Vella M, Muscat R. Brain symmetry index in healthy and stroke patients for assessment and prognosis. Stroke Res Treat. 2017;2017:8276136.

43. Malouin F, Richards CL, Desrosiers J, Doyon J. Bilateral slowing of mentally simulated actions after stroke. NeuroReport. 2004;15(8):1349-53.

44. Malouin F, Richards CL, Durand A. Normal aging and motor imagery vividness: implications for mental practice training in rehabilitation. Arch Phys Med Rehabil. 2010;91(7):1122-7.

45. Schuster C, Lussi A, Wirth B, Ettlin T. Two assessments to evaluate imagery ability: translation, test-retest reliability and concurrent validity of the German KVIQ and Imaprax. BMC Med Res Methodol. 2012;12:127.

46. Malouin F, Richards $\mathrm{CL}$, Jackson PL, Lafleur MF, Durand A, Doyon J. The kinesthetic and visual imagery questionnaire (KVIQ) for assessing motor imagery in persons with physical disabilities: a reliability and construct validity study. J Neurol Phys Ther. 2007:31(1):20-9.

47. Schuster C, Hilfiker R, Amft O, Scheidhauer A, Andrews B, Butler J, Kischka U, Ettlin $T$. Best practice for motor imageny: a systematic literature review on motor imagery training elements in five different disciplines. BMC Med. 2011;9:75.

48. Wondrusch C, Schuster-Amft C. A standardized motor imagery introduction program (MIII) for neuro-rehabilitation: development and evaluation. Front Hum Neurosci. 2013;7:477.

49. Carelli L, Solca F, Faini A, Meriggi P, Sangalli D, Cipresso P, Riva G, Ticozzi N, Ciammola A, Silani V, et al. Brain-computer Interface for clinical purposes: cognitive assessment and rehabilitation. Biomed Res Int. 2017;2017:1695290.

\section{Publisher's Note}

Springer Nature remains neutral with regard to jurisdictional claims in published maps and institutional affiliations.

Ready to submit your research? Choose BMC and benefit from:

- fast, convenient online submission

- thorough peer review by experienced researchers in your field

- rapid publication on acceptance

- support for research data, including large and complex data types

- gold Open Access which fosters wider collaboration and increased citations

- maximum visibility for your research: over $100 \mathrm{M}$ website views per year

At BMC, research is always in progress.

Learn more biomedcentral.com/submissions 